\title{
Asthma increases pulmonary thromboembolism risk: a nationwide population cohort study
}

\author{
Wei-Sheng Chung ${ }^{1,2,11}$, Cheng-Li Lin ${ }^{3}$, Feng-Ming Ho${ }^{4}$, Ruei-Yuan Li', \\ Fung-Chang Sung ${ }^{3,5}$, Chia-Hung $\mathrm{Kao}^{6,7,11}$ and Jun-Jun Yeh ${ }^{8,9,10}$
}

Affiliations: 'Dept of Internal Medicine, Taichung Hospital, Ministry of Health and Welfare, Taichung, ${ }^{2}$ Dept of Healthcare Administration, Central Taiwan University of Science and Technology, Taichung, ${ }^{3}$ Management Office for Health Data, China Medical University Hospital, Taichung, ${ }^{4}$ Dept of Internal Medicine, Taoyuan Hospital, Department of Health, Executive Yuan, Taoyuan, ${ }^{5}$ Dept of Public Health, China Medical University, Taichung, ${ }^{6}$ Graduate Institute of Clinical Medicine Science and School of Medicine, College of Medicine, China Medical University, Taichung, ${ }^{7}$ Dept of Nuclear Medicine and PET Center, China Medical University Hospital, Taichung, ${ }^{8}$ Ditmanson Medical Foundation Chia-Yi Christian Hospital, Chiayi, ${ }^{9}$ Chia Nan University of Pharmacy and Science, Tainan, and ${ }^{10}$ Meiho University, Pingtung, Taiwan. ${ }^{11}$ Both authors contributed equally.

Correspondence: J-J. Yeh, Department of Chest Medicine and Family Medicine, Ditmanson Medical Foundation Chia-Yi Christian Hospital, 539 Zhongxiao Road, Chiayi City, Taiwan 600, ROC.

E-mail: anvin.funlanamsa.hinet.net

ABSTRACT Studies on the association between asthma and pulmonary thromboembolism are considerably limited. We investigated whether pulmonary embolism is associated with asthma using a nationwide cohort study.

We identified 31356 patients with newly diagnosed asthma in 2002-2008 and 125157 individuals without asthma randomly selected from the general population, frequency matched by age, sex and index year using the National Health Insurance Research Database. Both cohorts were followed-up until the end of 2010 to measure the incidence of pulmonary embolism. Cox proportional hazards regression analysis was used to measure the hazard ratio of pulmonary embolism for the asthmatic cohort, compared with the nonasthmatic cohort.

We followed 186182 person-years for asthmatic patients and 743374 person-years for nonasthmatic subjects. The hazard ratio of pulmonary embolism was 3.24 for the asthmatic cohort, compared with the nonasthmatic cohort after adjusting for sex, age, comorbidities and oestrogen supplementation. The risk of developing pulmonary embolism significantly increased with the increased frequency of asthma exacerbation and hospitalisation.

This nationwide cohort study suggests that the risk of developing pulmonary embolism is significantly increased in asthmatic patients compared to the general population. Frequent asthma exacerbation and hospitalisation are significantly associated with pulmonary embolism risk.

@ERSpublications

Risk of pulmonary embolism in an asthmatic cohort was 3.24-fold compared with a nonasthmatic cohort http://ow.ly/rHEsF

For editorial comments see page 694.

Received: March 102013 | Accepted after revision: July 212013 | First published online: Aug 292013

Support statement: This work was supported by study projects at the China Medical University Hospital (Taichung, Taiwan) (DMR-100-076 and DMR-100-077), the Taiwan Department of Health Clinical Trial and Research Center of Excellence (DOH102-TD-B-111-004), the Taiwan Department of Health Cancer Research Center of Excellence (DOH102-TD-C-111-005) and the International Research-intensive Centers of Excellence in Taiwan (I-RiCE) (NSC1012911-I-002-303).

Conflict of interest: None declared.

Copyright (C)ERS 2014 


\section{Introduction}

Asthma has become a major cause of morbidity and mortality worldwide, and its prevalence has increased in the past 20 years [1]. Asthma is a chronic inflammatory disorder of the airways, which involves inflammatory cells and multiple mediators that result in characteristic pathophysiological changes. Chronic inflammation of the airways is associated with airway hyperresponsiveness, which leads to increased mucus plugs, recurrent attacks of breathlessness and wheezing. Asthma currently has no cure, except for symptomatic treatment.

Pulmonary thromboembolism is a blockage of the main pulmonary artery or one of its branches by a substance that has travelled from elsewhere in the body through the bloodstream. Pulmonary artery thromboses and pulmonary infarcts constitute pulmonary embolism, which may become a potentially fatal disorder with high mortality rates [2]. Although the major risk factors of pulmonary embolism are well recognised, the pathology often develops without any obvious precipitating factor. CusHMAN et al. [3] reported from a longitudinal investigation of thromboembolism aetiology that half of pulmonary embolism cases were idiopathic.

Studies have shown that chronic inflammatory diseases are connected with coagulation activation and increased pulmonary embolism risk [4-6]. Several studies have indicated pulmonary embolism prevalence and risk in patients with chronic obstructive pulmonary disease (COPD) [7-9]. Asthma and COPD are chronic inflammatory disorders of the airways. Recent studies have detected elevated concentrations of thrombin in sputum of asthmatic patients $[10,11]$. Further studies have also demonstrated that asthma is connected with prothrombotic factors and endothelial dysfunction in the development of atherothrombosis and pulmonary embolism $[12,13]$. However, asthma is not generally viewed as a risk factor for pulmonary embolism, although asthmatic patients may exhibit elevated concentrations of coagulation factors [10, 14]. A single study, in the Netherlands, has addressed the association between asthma and pulmonary embolism [15]. Therefore, we conducted a nationwide asthma cohort study to investigate whether asthma increases the risk of pulmonary embolism.

\section{Methods}

Data sources

Data analysed in this study were retrieved from the National Health Insurance Research Database (NHIRD), a database established and maintained by the National Health Research Institute (NHRI), which was released by the Bureau of National Health Insurance (BNHI). The BNHI provided the medical claims data after scrambling identification details, and this study was exempted by the institutional review board. The National Health Insurance programme in Taiwan has been operating since 1995; it covers 99\% of the island's 23.74 million population and was contracted by $97 \%$ of hospitals and clinics by the end of 2009 [16].

TABLE 1 Demographic characteristics, comorbidities and oestrogen supplementation in patients with and without asthma

\begin{tabular}{|c|c|c|c|}
\hline & Nonasthmatic subjects & Asthmatic patients & p-value \\
\hline Subjects & 125157 & 31356 & \\
\hline \multicolumn{4}{|l|}{ Sex } \\
\hline Female & $63896(51.1)$ & 15966 (50.9) & 0.67 \\
\hline Male & $61261(49.0)$ & $15390(49.1)$ & \\
\hline Age years & $38.9 \pm 25.7$ & $38.8 \pm 26.2$ & $0.26^{\#}$ \\
\hline \multicolumn{4}{|l|}{ Stratified age } \\
\hline$\leqslant 50$ years & 76580 (61.2) & 19225 (61.3) & 0.92 \\
\hline $50-65$ years & 22459 (17.9) & $5604(17.9)$ & \\
\hline$\geqslant 65$ years & $26118(20.9)$ & $6527(20.8)$ & \\
\hline \multicolumn{4}{|l|}{ Comorbidity } \\
\hline Atrial fibrillation & $731(0.58)$ & $329(1.05)$ & $<0.0001$ \\
\hline Diabetes & 11370 (9.08) & $3528(11.3)$ & $<0.0001$ \\
\hline Hyperlipidaemia & $15065(12.0)$ & $5036(16.1)$ & $<0.0001$ \\
\hline CVD & $8902(7.11)$ & $3019(9.63)$ & $<0.0001$ \\
\hline Heart failure & $2073(1.66)$ & $1334(4.25)$ & $<0.0001$ \\
\hline Lower leg fracture or surgery & $1644(1.31)$ & $528(1.68)$ & $<0.0001$ \\
\hline Cancer & 4005 (3.20) & $1171(3.73)$ & $<0.0001$ \\
\hline Oestrogen supplementation & $17372(13.9)$ & $5252(16.8)$ & $<0.0001$ \\
\hline
\end{tabular}

Data are presented as $\mathrm{n}, \mathrm{n}(\%)$ or mean $\pm \mathrm{SD}$, unless otherwise stated. $\mathrm{p}$-values were calculated using the Chi-squared test, unless otherwise stated. CVD: cerebrovascular disease. ${ }^{\#}$ : two-sample t-test. 


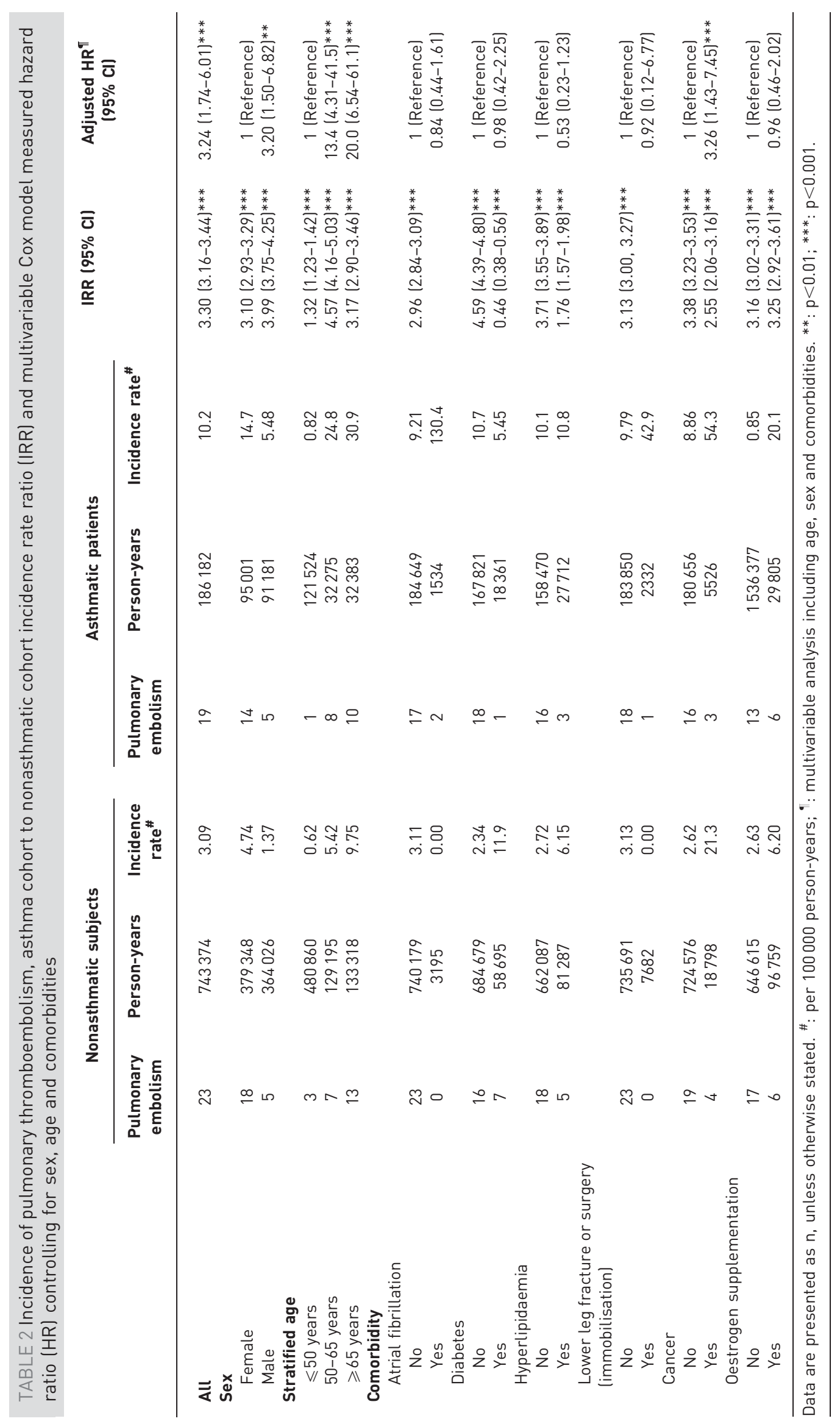


TABLE 3 Hazard ratio (HR) of pulmonary thromboembolism risk associated with the number of emergency room visits and admissions due to asthma exacerbation

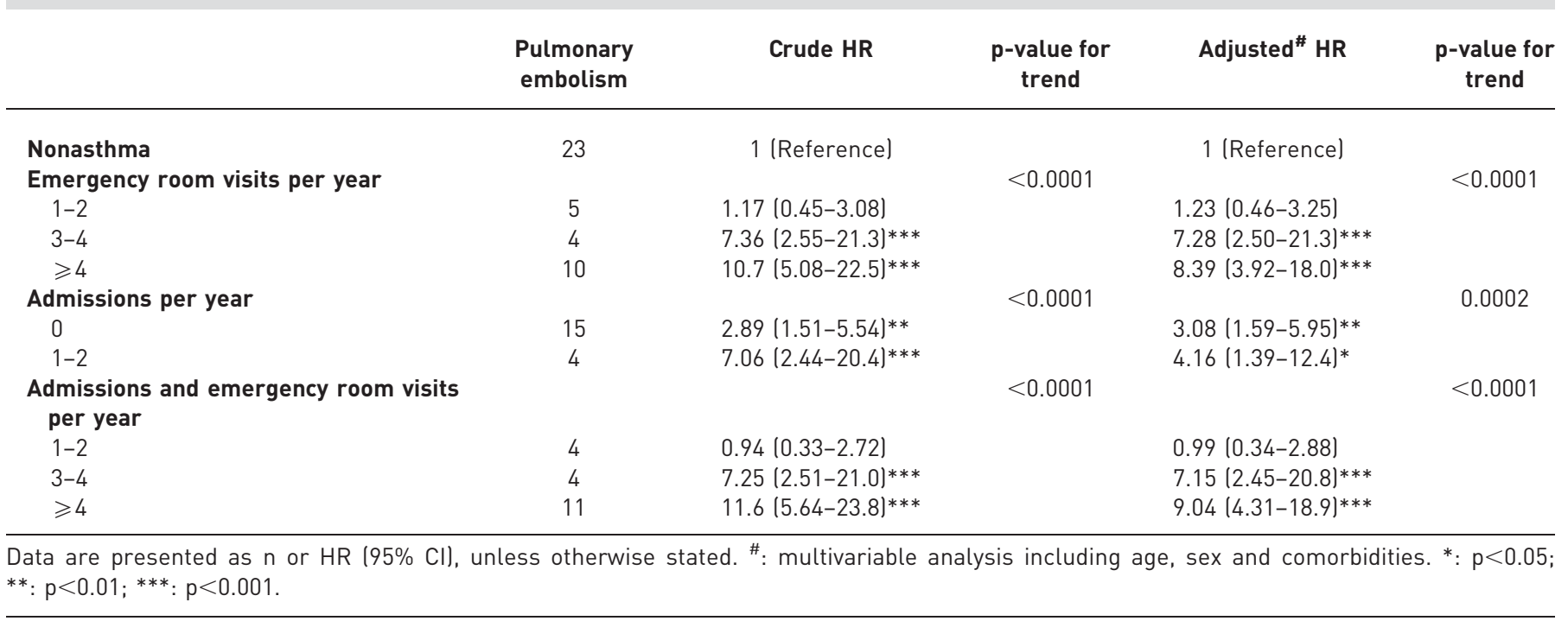

We used a systemic sampling of the patient data of 1 million participants from all insured beneficiaries, which was released by the NHRI as the Longitudinal Health Insurance Database (LHID). The NHRI reported no significant age and sex differences between the LHID and all insurants. CHENG et al. [17] and KANG et al. [18] have demonstrated the accuracy and high validity of diagnoses in the NHIRD. The International Classification of Disease 9th revision Clinical Modification (ICD-9-CM) was used for the diagnostic codes. This study was approved by the institutional review board of China Medical University in central Taiwan (CMU-REC-101-012).

\section{Study patients}

Patients newly diagnosed with bronchial asthma (ICD-9-CM code 4930-4939) in 2002-2008 were identified from ambulatory case visits or admission records, and designated the asthmatic cohort. The index date for patients with bronchial asthma diagnosis was the date of their first medical visit. Patients with a history of pulmonary embolism (ICD-9-CM code 415.1) or deep vein thrombosis (DVT) (ICD-9-CM code 453.8) before the index date, or with incomplete age or sex information, were excluded. For each asthmatic case identified, four insured people without a history of asthma or pulmonary embolism were randomly selected, frequency matched in the same year, and designated as the nonasthma controls. Patients and controls were matched for age (each 5-year span) and sex.

\section{Outcome measures}

All participants were observed to measure the incidence of pulmonary embolism incidence (excluding iatrogenic incidence (ICD-9-CM code 415.11)) until the end of 2010 or censored for death, emigration or discontinuation of enrolment in the NHIRD. The baseline history of comorbidity for each participant was identified, including atrial fibrillation (ICD-9-CM code 427.31), diabetes (ICD-9-CM code 250), hyperlipidaemia (ICD-9-CM code 272), cerebrovascular disease (CVD; ICD-9-CM code 430-438), heart failure (ICD-9-CM code 428), lower leg fracture or surgery (ICD-9-CM code 820, 821; 823; 81.51, 81.52, 81.53 or 81.54 ) and cancer (ICD-9-CM code 140-208). Lower leg fracture or surgery (immobilisation), as well as medication such as oestrogen supplementation, which could affect the development of pulmonary embolism, were also included as covariates in the analysis.

\section{Statistical analysis}

The distributions of categorical demographic variables and comorbidities were compared between asthmatic patients and nonasthmatic cohorts, and the differences were examined using a Chi-squared test. The mean age of both cohorts was measured and tested using the t-test. Similarly, incidence densities by demographic variables and comorbidity were calculated for each cohort. The asthma to nonasthma rate ratio for pulmonary embolism was calculated with incidence rate ratios and $95 \%$ confidence interval (CI) for each variable. The rate ratio was determined using Poisson regression. Multivariable Cox proportionalhazards regression was used to assess the risk of developing pulmonary embolism associated with asthma after adjusting for the variables significantly related to pulmonary embolism. Hazard ratios (HR) and 95\% 
TABLE 4 Incidence rate ratio (IRR) and hazard ratio (HR) of pulmonary thromboembolism events by follow-up years

\begin{tabular}{|c|c|c|c|c|c|c|c|c|}
\hline \multirow[t]{2}{*}{ Follow-up } & \multicolumn{3}{|c|}{ Nonasthmatic cohort } & \multicolumn{3}{|c|}{ Asthma cohort } & \multirow{2}{*}{$\begin{array}{c}\text { IRR } \\
(95 \% \mathrm{CI})\end{array}$} & \multirow{2}{*}{$\begin{array}{c}\text { Adjusted HR } \\
(95 \% \mathrm{CI})\end{array}$} \\
\hline & $\begin{array}{l}\text { Pulmonary } \\
\text { embolism }\end{array}$ & Person-years & Rate $^{\#}$ & $\begin{array}{l}\text { Pulmonary } \\
\text { embolism }\end{array}$ & Person-years & Rate $^{\#}$ & & \\
\hline$\leqslant 5$ years & 18 & 560371 & 3.21 & 16 & 140209 & 11.4 & $\begin{array}{c}3.55 \\
(3.40-3.71)^{* * *}\end{array}$ & $\begin{array}{c}3.38 \\
(1.70-6.74)^{* * *}\end{array}$ \\
\hline$>5$ years & 5 & 183003 & 2.73 & 3 & 45973 & 6.53 & $\begin{array}{c}2.39 \\
(2.26-2.53)^{* * *}\end{array}$ & $\begin{array}{c}2.43 \\
(0.57-10.4)\end{array}$ \\
\hline
\end{tabular}

Data are presented as $n$, unless otherwise stated. " : incidence rate per 100000 person-years; " : multivariable analysis including age, sex and comorbidities. ${ }^{* * *}: \mathrm{p}<0.001$.

CI were calculated using this model. The Cox model was also used to estimate the HR of pulmonary embolism associated with the cumulative frequency of emergency department visits or admission due to asthma, compared to the nonasthmatic cohort. We further assessed the role of asthma duration using timedependent covariates ( $\leqslant 5$ years and $>5$ years since asthma diagnosis). The cumulative incidence of pulmonary embolism between the asthmatic patients and the nonasthmatic cohort were estimated using the Kaplan-Meier method, and the differences were assessed using a log-rank test. All statistical analyses were performed using SAS version 9.2 (SAS Institute Inc., Cary, NC, USA), and the Kaplan-Meier survival curve was plotted using Stata 11.0 (StataCorp, College Station, TX, USA). The level of statistical significance was set at 0.05 .

\section{Results}

Demographic characteristics of the study participants

31356 asthmatic patients and 125157 nonasthmatic controls were enrolled to our study after excluding ineligible participants. Table 1 shows a comparison of the demographic characteristics and comorbidities of the asthmatic patients and the nonasthmatic cohort. Most participants were aged $\leqslant 50$ years $(61.2 \%$ of asthmatics and $61.1 \%$ of nonasthmatics). The asthmatic cohort had a greater prevalence of atrial fibrillation, diabetes, hyperlipidaemia, CVD, heart failure, lower leg fracture or surgery (immobilisation), cancer and oestrogen supplementation at baseline $(\mathrm{p}<0.05)$.

\section{The incidence rate and HR of pulmonary embolism stratified by sex, age and comorbidity between two cohorts}

The overall incidence rate of pulmonary embolism showed a 3.30-fold increase in asthmatic patients compared to the nonasthmatic cohort (10.2 versus 3.09 per 100000 person-years). The adjusted overall HR of pulmonary embolism in asthmatic patients was 3.24 (95\% CI 1.74-6.01) (table 2) compared with the nonasthmatic cohort, after controlling for sex, age, comorbidities and oestrogen use. Sex-specific analysis showed the incidence rate of males and females with asthma at 5.48 and 14.7 per 100000 personyears, respectively; higher than that in the nonasthmatic cohort (1.37 and 4.74 per 100000 person-years, respectively). Males had a 3.20-fold increased risk of pulmonary embolism development compared to females after adjusting for age, asthma and comorbidities. An age-specific analysis showed that asthmatic patients had a higher incidence rate of pulmonary embolism development than the nonasthmatic cohort in all age groups, and that the pulmonary embolism incidence rate increased with age. The incidence rates of pulmonary embolism were significantly higher in the asthmatic cohort than in the nonasthmatic cohort, except for patients with diabetes. Patients with cancer were at a higher risk of pulmonary embolism than patients who did not have cancer (HR 3.26, 95\% CI 1.43-7.45).

\section{Relationship between the number of emergency room visits and admissions and risk of pulmonary embolism}

The association between the average number of emergency room visits and admissions because of asthma exacerbation and pulmonary embolism development was measured using cumulative frequency (table 3). The HR increased with an increased number of emergency room visits and admissions. Compared to the nonasthmatic cohort, the adjusted HR (95\% CI) increased with the number of emergency room visits and admissions because of asthma exacerbation, from 0.99 (0.34-2.88) for those having two or fewer visits, up to 9.04 (4.31-18.9) for those having four and more visits $(\mathrm{p}<0.0001$ for trend $)$. 


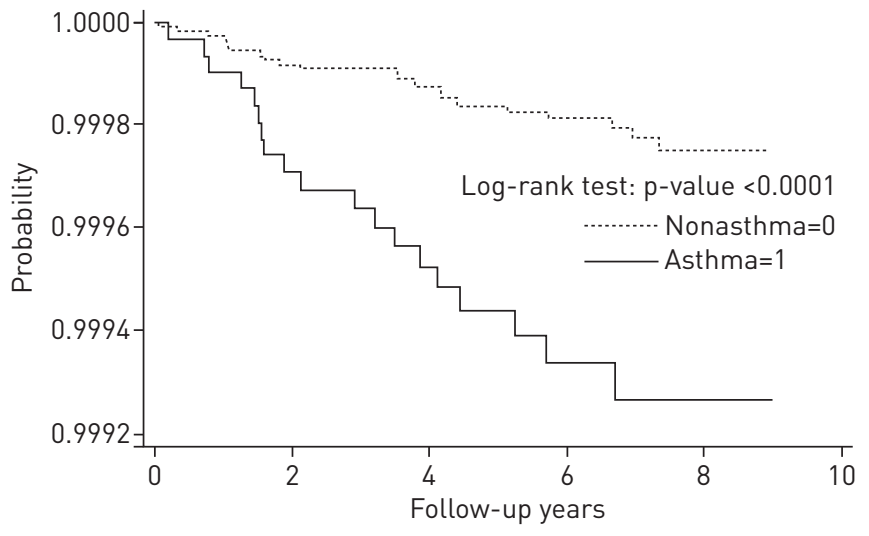

FIGURE 1 Kaplan-Meier analysis comparing probabilities of pulmonary thromboembolism between asthmatic patients and the nonasthmatic cohort.

Trends of pulmonary embolism events by stratified follow-up years

During the first 5 years after asthma diagnosis, the pulmonary embolism incidence rate was higher in the asthmatic patients than in the nonasthmatic cohort (11.4 versus 3.21 per 100000 person-years), with an adjusted HR (95\% CI) of 3.38 (1.70-6.74) (table 4). Kaplan-Meier survival analysis showed that patients with asthma had significantly higher pulmonary embolism rates than the nonasthmatic cohort (fig. 1).

\section{Discussion}

This is the first study to investigate whether an Asian population with asthma have an increased risk of developing pulmonary embolism through a longitudinal population-based cohort study. Our study shows that asthmatic patients have a 3.24-fold increased risk of pulmonary embolism development than the general population after adjusting for age, sex and comorbidities. Only one Western study [15] indicated asthma with increased pulmonary embolism risk and our finding is consistent with theirs. Several studies have also shown an increased prevalence and pulmonary embolism risk in COPD patients [6-9].

Although several potential mechanisms exist, the nature of the association between asthma and pulmonary embolism remains unclear. Elevated concentrations of thrombin have been found in the sputum and bronchoalveolar lavage of asthmatic patients, further supporting the existence of local coagulation activation in asthma $[11,19]$. Thrombin may play a role in inflammation and remodelling, in addition to its central role of haemostasis. The airways represent a body compartment in which coagulation may be initiated locally [20]. Several studies have also indicated that vascular endothelial dysfunction results from reduced activation of endothelial nitric oxide in asthmatic patients [12, 13].

MAJOOR et al. [15] first showed the relationship between asthma and pulmonary embolism in a retrospective study, in which the study participants were enrolled from three Dutch asthma outpatient clinics. Our study conducted a nationwide population-based cohort study from the NHIRD and demonstrated asthma with an increased risk of subsequent pulmonary embolism development.

Asthmatic patients have an increased risk of subsequent pulmonary embolism development in all groups. Females have a higher incidence rate of pulmonary embolism than males. However, males have a significantly higher adjusted hazard ratio of subsequent pulmonary embolism development after controlling for age and comorbidities. This finding is consistent with previous studies [21, 22].

The pulmonary embolism incidence rate significantly increased with age in both sexes, which is also proven after adjusting for the covariate. This finding is compatible with previous studies [3, 23]. Venous thromboembolism is predominantly a disease of older people. Incidence rates and risks increase exponenetially for both males and females and for both pulmonary embolism and DVT.

The Global Initiative for Asthma has recommended asthma care based on the clinical control status of uncontrolled, partly controlled and good control, instead of asthma severity [1]. No study has investigated the relationship between the number of asthma exacerbations and related hospital admissions to the risk of pulmonary embolism development. Our study shows that the risk of developing pulmonary embolism increased with the number of asthma exacerbations, emergency room visits and admissions. This finding suggests poor control as an important factor for pulmonary embolism in asthmatic patients. The mechanism by which the number of asthma exacerbations, emergency room visits and admissions may predispose pulmonary embolism seems complex. Inflammation may alter the balance between procoagulant and fibrinoytic activities because inflammation and coagulation stimulate each other. Patients with asthma exacerbation present with tachypnoea and hypoxaemia, which may dehydrate the body and increase the 
likelihood of developing pulmonary embolism. According to the trends of pulmonary embolism event risk in asthmatic patients, pulmonary embolism development significantly increased within 5 years of asthma follow-up.

The strength of this study is that it provides a nationwide population-based cohort longitudinal study on the risk of pulmonary embolism development in people with asthma. These findings can be extended to the general population. However, several limitations must be considered when interpreting these findings. The NHIRD does not provide detailed lifestyle information, such as smoking, body mass index and physical activity, which are all potential confounding factors for this study. Secondly, the lack of corticosteroid doses and other drugs affecting blood coagulation may present another limitation. However, whether the use of corticosteroids contributes to a hypercoagulable state is controversial [24].

Our nationwide study of 31356 asthmatic patients with 186182 person-years of follow-up shows that asthmatic patients have a 3.24-fold increased risk of developing pulmonary embolism compared to the general population. These findings highlight the importance of clinician awareness of potential pulmonary embolism development among asthmatic patients.

\section{References}

Global Strategy for Asthma Management and Prevention. Global Initiative for Asthma. 2012 www.ginasthma.org

2 Janata K, Holzer M, Domanovits H, et al. Mortality of patients with pulmonary embolism. Wien Klin Wochenschr 2002; 114: 766-772.

3 Cushman M, Tsai AW, White RH, et al. Deep vein thrombosis and pulmonary embolism in two cohorts: the longitudinal investigation of thromboembolism etiology. Am J Med 2004; 117: 19-25.

4 Choi HK, Rho YH, Zhu Y, et al. The risk of pulmonary embolism and deep vein thrombosis in rheumatoid arthritis: a UK population-based outpatient cohort study. Ann Rheum Dis 2013; 72: 1182-1187.

5 Grainge MJ, West J, Card TR. Venous thromboembolism during active disease and remission in inflammatory bowel disease: a cohort study. Lancet 2010; 375: 657-663.

6 Rizkallah J, Man SF, Sin DD. Prevalence of pulmonary embolism in acute exacerbations of COPD: a systematic review and metaanalysis. Chest 2009; 135: 786-793.

7 Carson JL, Terrin ML, Duff A, et al. Pulmonary embolism and mortality in patients with COPD. Chest 1996; 110: 1212-1219.

8 Tillie-Leblond I, Marquette CH, Perez T, et al. Pulmonary embolism in patients with unexplained exacerbation of chronic obstructive pulmonary disease: prevalence and risk factors. Ann Intern Med 2006; 144: 390-396.

9 Bertoletti L, Quenet S, Mismetti P, et al. Clinical presentation and outcome of venous thromboembolism in COPD. Eur Respir J 2012; 39: 862-868.

10 Kanazawa H, Yoshikawa T. Up-regulation of thrombin activity induced by vascular endothelial growth factor in asthmatic airways. Chest 2007; 132: 1169-1174.

11 Schouten M, van de Pol MA, Levi M, et al. Early activation of coagulation after allergen challenge in patients with allergic asthma. J Thromb Haemost 2009; 7: 1592-1594.

12 Yildiz P, Oflaz H, Cine N, et al. Endothelial dysfunction in patients with asthma: the role of polymorphisms of ACE and endothelial NOS genes. J Asthma 2004; 41: 159-166.

13 Wanner A, Mendes ES. Airway endothelial dysfunction in asthma and chronic obstructive pulmonary disease: a challenge for future research. Am J Respir Crit Care Med 2010; 182: 1344-1351.

14 de Boer JD, Majoor CJ, van't Veer C, et al. Asthma and coagulation. Blood 2012; 119: 3236-3244.

15 Majoor CJ, Kamphuisen PW, Zwinderman AH, et al. Risk of deep vein thrombosis and pulmonary embolism in asthma. Eur Respir J 2013; 42: 655-661.

16 Cheng T-M. Taiwan's national health insurance system: high value for the dollar. In: Okma KGH, Crivelli L, Klein R, eds. Six Countries, Six Reform Models: the Health Reform Experience of Israel, the Netherlands, New Zealand, Singapore, Switzerland and Taiwan. New Jersey, World Scientific, 2009; pp. 171-204.

17 Cheng CL, Kao YH, Lin SJ, et al. Validation of the National Health Insurance Research Database with ischemic stroke cases in Taiwan. Pharmacoepidemiol Drug Saf 2011; 20: 236-242.

18 Kang JH, Chen YH, Lin HC. Comorbidity profiles among patients with ankylosing spondylitis: a nationwide population-based study. Ann Rheum Dis 2010; 69: 1165-1168.

19 Terada M, Kelly EA, Jarjour NN. Increased thrombin activity after allergen challenge: a potential link to airway remodeling? Am J Respir Crit Care Med 2004; 169: 373-377.

20 Levi M, Schultz MJ, Rijneveld AW, et al. Bronchoalveolar coagulation and fibrinolysis in endotoxemia and pneumonia. Crit Care Med 2003; 31: Suppl. 4, S238-S242.

21 Silverstein MD, Heit JA, Mohr DN, et al. Trends in the incidence of deep vein thrombosis and pulmonary embolism: a 25-year population-based study. Arch Intern Med 1998; 158: 585-593.

22 Lapostolle F, Le Toumelin P, Chassery C, et al. Gender as a risk factor for pulmonary embolism after air travel. Thromb Haemost 2009; 102: 1165-1168.

23 Heit JA. The epidemiology of venous thromboembolism in the community. Arterioscler Thromb Vasc Biol 2008; 28: 370-372.

24 van Zaane B, Nur E, Squizzato A, et al. Systematic review on the effect of glucocorticoid use on procoagulant, anticoagulant and fibrinolytic factors. J Thromb Haemost 2010; 8: 2483-2493. 